\title{
BMJ Open Prenatal diagnosis and prevalence of critical congenital heart defects: an international retrospective cohort study
}

Marian K Bakker, ${ }^{01}$ Jorieke E H Bergman, ${ }^{1}$ Sergey Krikov, ${ }^{2}$ Emmanuelle Amar, ${ }^{3}$ Guido Cocchi, ${ }^{4}$ Janet Cragan, ${ }^{5}$ Hermien E K de Walle, ${ }^{1}$ Miriam Gatt, ${ }^{6}$ Boris Groisman, ${ }^{7}$ Shiliang Liu, ${ }^{8}$ Wendy N Nembhard, ${ }^{9}$ Anna Pierini, ${ }^{10}$ Anke Rissmann, ${ }^{11}$ Shanthi Chidambarathanu, ${ }^{12}$ Antonin Sipek Jr, ${ }^{13}$ Elena Szabova, ${ }^{14}$ Giovanna Tagliabue, ${ }^{15}$ David Tucker, ${ }^{16}$ Pierpaolo Mastroiacovo, ${ }^{17}$ Lorenzo D Botto ${ }^{2,17}$

To cite: Bakker MK Bergman JEH, Krikov S, et al. Prenatal diagnosis and prevalence of critical congenital heart defects: an international retrospective cohort study. BMJ Open 2019;9:e028139. doi:10.1136/ bmjopen-2018-028139

- Prepublication history and additional material for this paper are available online. To view these files, please visit the journal online (http://dx.doi. org/10.1136/bmjopen-2018028139).

Received 5 December 2018 Revised 10 May 2019

Accepted 11 June 2019

Check for updates

(C) Author(s) (or their employer(s)) 2019. Re-use permitted under CC BY. Published by BMJ.

For numbered affiliations see end of article.

Correspondence to Dr Marian K Bakker; m.k.bakker@umcg.nl

\section{ABSTRACT}

Objectives To assess international trends and patterns of prenatal diagnosis of critical congenital heart defects (CCHDs) and their relation to total and live birth CCHD prevalence and mortality.

Setting Fifteen birth defect surveillance programmes that participate in the International Clearinghouse for Birth Defects Surveillance and Research from 12 countries in Europe, North and South America and Asia.

Participants Live births, stillbirths and elective terminations of pregnancy for fetal anomaly diagnosed with 1 of 12 selected CCHD, ascertained by the 15 programmes for delivery years 2000 to 2014 .

Results 18243 CCHD cases were reported among 8847 081 births. The median total prevalence was 19.1 per 10000 births but varied threefold between programmes from 10.1 to 31.0 per 10000 . CCHD were prenatally detected for at least $50 \%$ of the cases in one-third of the programmes. However, prenatal detection varied from $13 \%$ in Slovak Republic to $87 \%$ in some areas in France. Prenatal detection was consistently high for hypoplastic left heart syndrome ( $64 \%$ overall) and was lowest for total anomalous pulmonary venous return (28\% overall). Surveillance programmes in countries that do not legally permit terminations of pregnancy tended to have higher live birth prevalence of CCHD. Most programmes showed an increasing trend in prenatally diagnosed CCHD cases. Discussion and conclusions Prenatal detection already accounts for $50 \%$ or more of CCHD detected in many programmes and is increasing. Local policies and access likely account for the wide variability of reported occurrence and prenatal diagnosis. Detection rates are high especially for CCHD that are more easily diagnosed on a standard obstetric four-chamber ultrasound or for fetuses that have extracardiac anomalies. These ongoing trends in prenatal diagnosis, potentially in combination with newborn pulse oximetry, are likely to modify the epidemiology and clinical outcomes of CCHD in the near future.

\section{INTRODUCTION}

Congenital heart defects (CHDs) are among the most common birth defects, affecting approximately 1 in 100 births. $^{1}{ }^{2}$ About

\section{Strengths and limitations of this study}

- This retrospective cohort study includes a large sample of more than 18000 cases with critical congenital heart defects from 15 birth defect surveillance programmes from Europe, North and South America and Asia.

- The programmes come from areas with different policies regarding prenatal screening and diagnosis and therefore allow a wider view of factors related to prevalence, ascertainment and prenatal diagnosis.

- The individual case records were centrally reviewed by clinicians with expertise in genetics and paediatric cardiology in order to harmonise diagnoses and clinical classification.

- The quality and completeness of the data depend on the programme's methods related to data collection, coding and classification.

- Details on the severity of each case were not available.

$20 \%-25 \%$ of CHD, or about 1 in 500 births, have been described as critical congenital heart defects (CCHDs) because they require urgent and significant medical and surgical care to ensure survival. ${ }^{13}$ CCHD represent a significant clinical and public health challenge. In lower income countries, where complex health resources are the scarcest, CCHD are associated with very high mortality. In high-income countries, including North America and Europe, CCHDs are associated with lifelong morbidities and, for healthcare systems, with some of the leading drivers for paediatric in-hospital care costs. ${ }^{45}$

Treatment and outcomes of CCHD have improved dramatically over the last decades. ${ }^{6-9}$ A major part of the treatment strategy is to identify CCHD as early as possible, so that a management plan can be agreed on and put in place prior to the baby presenting acutely 
and often in cardiac failure. ${ }^{10-14}$ Prenatal diagnosis and newborn screening are two such early detection strategies, with prenatal diagnosis allowing for more deliberate management planning with family and care providers.

Prenatal detection of CCHD depends on several factors, including technology (the availability of adequate equipment), sonographer skills (CCHD detection requires more experience than the standard prenatal anatomic scan), screening policies and access to prenatal screening services (location and costs). ${ }^{15} 16$ Because these factors vary by country, within a country, and over time, as services and policies evolve, so will the rate and impact of prenatal diagnosis of CCHD. In turn, the rate of prenatal diagnosis can have multiple consequences on the pattern, trends and outcomes of CCHD in a given population. Through earlier detection, prenatal diagnosis will improve overall ascertainment of CCHD by the time of birth, which could be reflected in more accurate estimates of prevalence at birth by birth registries. This in turn can improve longitudinal population-based surveillance of CCHD-related outcomes through registry or linkage studies. Prenatal detection may also be associated with elective terminations of pregnancy for fetal anomaly (TOPFA), possibly reducing the live birth (LB) prevalence of CCHD and changing the overall pattern of CHD in the population. ${ }^{17}$ Thus, prenatal diagnosis of CCHD has the potential of changing the epidemiology and public health impact of CCHD in complex ways. In this study, we examined the changing trends of prenatal diagnosis of CCHD and their impact on CCHD birth prevalence and mortality in a geographically diverse set of programmes that participate in the International Clearinghouse for Birth Defects Surveillance and Research (ICBDSR).

\section{METHODS}

\section{Study design and contributing programmes}

This retrospective cohort study is based on data from 15 birth defect surveillance programmes (table 1) that are members of the ICBDSR. The ICBDSR is an international network of birth defects surveillance and research programmes, whose mission is collaborative surveillance of birth defects and research into their causes and outcomes (www.icbdsr.org). The 15 programmes represent 12 countries from Europe, North America, South America and Asia. Participating programmes had to be able to provide case-level data with specific diagnoses for CHD and extracardiac malformations for at least two birth years. Most contributing programmes are population based, while the remainder are hospital based. The programme from India is hospital based and a solely prenatal programme, meaning that only cases that are prenatally diagnosed

Table 1 Selected geographic, registration procedure and policy characteristics of participating surveillance programmes, International Clearinghouse for Birth Defects Surveillance and Research (ICBDSR) Critical Congenital Heart Defects (CCHD) Prenatal Diagnosis study 2000-2014

\begin{tabular}{|c|c|c|c|c|c|c|}
\hline Country & Area & $\begin{array}{l}\text { Type of } \\
\text { programme* }\end{array}$ & $\begin{array}{l}\text { Ascertainment } \\
\text { period }\end{array}$ & $\begin{array}{l}\text { TOPFA } \\
\text { legal }\end{array}$ & $\begin{array}{l}\text { Stillbirth definition } \\
\text { for study }\end{array}$ & $\begin{array}{l}\text { Birth years } \\
\text { contributed } \\
\text { to study }\end{array}$ \\
\hline UK & Wales & $P$ & 18 years & Yes & $\geq 24$ WGA & 2001-2012 \\
\hline Germany & Saxony Anhalt & $\mathrm{P}$ & 1 year & Yes & $\geq 500 \mathrm{~g}$ & 2001-2012 \\
\hline The Netherlands & Northern & $P$ & 10 years & Yes & $\geq 24$ WGA & 2001-2012 \\
\hline France & Rhone Alpes & $\mathrm{P}$ & 18years & Yes & $\geq 20$ WGA & 2006-2012 \\
\hline Italy & Emilia Romagna & $P$ & 1 year & Yes & $\geq 20$ WGA & 2001-2012 \\
\hline Italy & Lombardy & $\mathrm{P}$ & 6 years & Yes & $\geq 23$ WGA & 2009-2010 \\
\hline Italy & Tuscany & $P$ & 1 year & Yes & $\geq 20$ WGA & 2001-2012 \\
\hline Malta & National & $\mathrm{P}$ & 1 year & No & $\geq 22 \mathrm{WGA}$ or $>500 \mathrm{~g}$ & 2001-2012 \\
\hline Czech Republic & National & $\mathrm{P}$ & 15 years & Yes & $\geq 28 \mathrm{WGA}$ or $>1000 \mathrm{~g}$ & 2000-2013 \\
\hline Slovak Republic & National & $\mathrm{P}$ & Hospital discharge & Yes & $\geq 1000 \mathrm{~g}$ & 2001-2012 \\
\hline Canada & National & $\mathrm{P}$ & 1 year & Yes & $\begin{array}{l}\geq 20 \text { WGA or }>500 \mathrm{~g} \\
\text { (or }>22 \text { WGA if birth } \\
\text { weight is unknown) }\end{array}$ & 2004-2014 \\
\hline USA & Arkansas & $P$ & 2 years & Yes & $\geq 20 W G A$ or $>350 \mathrm{~g}$ & 2001-2010 \\
\hline USA & Atlanta & $P$ & 6 years & Yes & $\geq 20$ WGA & $2001-2008$ \\
\hline Argentina & National & $\mathrm{H}$ & Hospital discharge & No & $\geq 500 \mathrm{~g}$ & 2013-2014 \\
\hline India & Chennai & $\mathrm{H}$ & Prenatal only & Yes & n.a. & 2008-2012 \\
\hline
\end{tabular}

*Type of programme: $\mathrm{H}$ : hospital based; P: population based.

†Data for Quebec not included (not available).

CCHDs, critical congenital heart defects; n.a, not applicable (live fetuses only, prenatal screening programme); TOPFA, termination of pregnancy for fetal anomaly; WGA, weeks of gestational age. 
within the contributing hospitals are registered within the programme. The other programmes include both prenatally and postnatally diagnosed cases.

\section{Data contributed}

The study included cases (LBs, stillbirths (SBs) and TOPFAs, depending on programme) with 1 of 12 types of CCHD: hypoplastic left heart syndrome (HLHS), coarctation of the aorta (COA), aortic valve stenosis (AoS), tetralogy of Fallot (TOF), d-transposition of great arteries (DTGA), double outlet right ventricle, persistent truncus arteriosus (PTA), interrupted aortic arch (IAA), pulmonary valve atresia with intact ventricular septum, tricuspid valve atresia/hypoplastic right heart (TriA/HRH), single ventricle (SV) and total anomalous pulmonary venous return (TAPVR). These CCHDs are identifiable prenatally through ultrasound either by a four-chamber view or an outflow tract view. The programmes review medical records and abstract clinical information including the diagnoses which, depending on local practices, are made by obstetricians or paediatric cardiologist dependings. The diagnoses are coded and classified by trained registry staff. For each case with 1 of the 12 selected CCHD, programmes provided the following key information: type of CCHD (International Classification of Disease, 9th Revision - Clinical Modification (ICD-9-CM) or International Classification of Disease, 10th Revision - Clinical Modification (ICD-10-CM) code plus verbatim description (if available), timing of diagnosis (prenatal versus postnatal), pregnancy outcome (LB, SB and TOPFA), presence of extracardiac anomalies (structural malformations or syndrome diagnoses, as ICD code plus verbatim description) and, for LBs, survival up to 1year of age. Cases with an end-of-pregnancy date (delivery or termination of pregnancy) between 2000 and 2014 were included in the study. Most programmes provided data for the time period from 2001 to 2012. Italy-Lombardy provided data on 2009 and 2010 and Argentina provided data on birth years 2013-2014. For the years for which they provided cases, programmes also provided corresponding yearly denominator data, including total number of LBs and total number of SBs.

For cases with more than one CCHD diagnoses, one clinical geneticist with specific expertise in paediatric cardiology (LDB) developed a structured hierarchical process to assign a single main CCHD diagnosis (for details, see the appendix in the online supplementary material). In addition, two clinical geneticists (LDB and JEHB) reviewed all cases with extracardiac or syndrome diagnoses to classify the case either as isolated, with multiple congenital anomalies (MCA) or genetic/ syndromic. MCA was defined as any combination of congenital anomalies (cardiac plus one or more extracardiac anomalies) without a recognised underlying cause (genetic or teratogenic) and not constituting a sequence.

Along with case data, programmes also completed a short questionnaire on local practices and policies related to prenatal diagnosis and pregnancy termination.
With the exception of Argentina and Malta, termination of pregnancy was legal in the areas covered by contributing programmes (table 1). In all regions covered by the programmes, ultrasound scans are performed as part of standard obstetric care, including a scan around 18-20 weeks. These scans, depending on local healthcare systems, can be free of charge. In the Netherlands, a routine screening programme for congenital anomalies is offered since 2007, while in Argentina, screening is part of standard obstetric care but depends on availability of technology.

\section{Analyses}

The analyses focused on prevalence, time of detection, clinical presentation and survival. Because some programmes contributed considerably more cases than others, and because a main goal of the study was to examine variations across programmes and countries, the findings are presented primarily by programme rather than in the aggregate. We calculated total prevalence and LB prevalence, with $95 \%$ CI computed based on the normal distribution. Total prevalence was calculated as total cases $(\mathrm{LB}+\mathrm{SB}+\mathrm{TOPFA})$ divided by births $(\mathrm{LB}+\mathrm{SB})$, expressed per 10000 births. LB prevalence was calculated as number of live born cases divided by total number of LBs per 10000 births. For programmes that contributed more than 2 years of data, we examined time trends in total prevalence and used the $\chi^{2}$ test for trend. Timing of detection of the CCHD (prenatal vs postnatal) was examined by programme, by CCHD type and by clinical presentation (isolated, MCA and genetic/syndromic). The proportion prenatally diagnosed over time was also examined for trends ( $\chi^{2}$ test for trend). Analyses were performed in Excel (Microsoft Office Professional plus 2010) and IBM SPSS Statistics for Windows, V.23.0. Each programme has local approved procedures for ethics approval, and because this study was done using deidentified data, no additional ethics committee approval was required.

\section{Patient and public involvement}

No patients were involved in setting the research question or the outcome measures, nor were they involved in developing plans for implementation of the study. No patients were asked to advise on interpretation or writing up of results. There are no plans to disseminate the results of the research to study participants or the relevant patient community.

\section{RESULTS}

\section{Prevalence}

Programmes ascertained 18243 CCHD cases among 8847081 births. The median prevalence was 19.1 per 10000 births or 1 in 524 births (IQR: 18.2-22.2 per 10000 births). The highest total prevalence was observed in the Czech Republic (30.9 per 10000 births) and the lowest in Slovak Republic and Argentina 
Table 2 Total prevalence of CCHD types per 10000 births, International Clearinghouse for Birth Defects Surveillance and Research (ICBDSR) Critical Congenital Heart Defects (CCHD) Prenatal Diagnosis study 2000-2014*

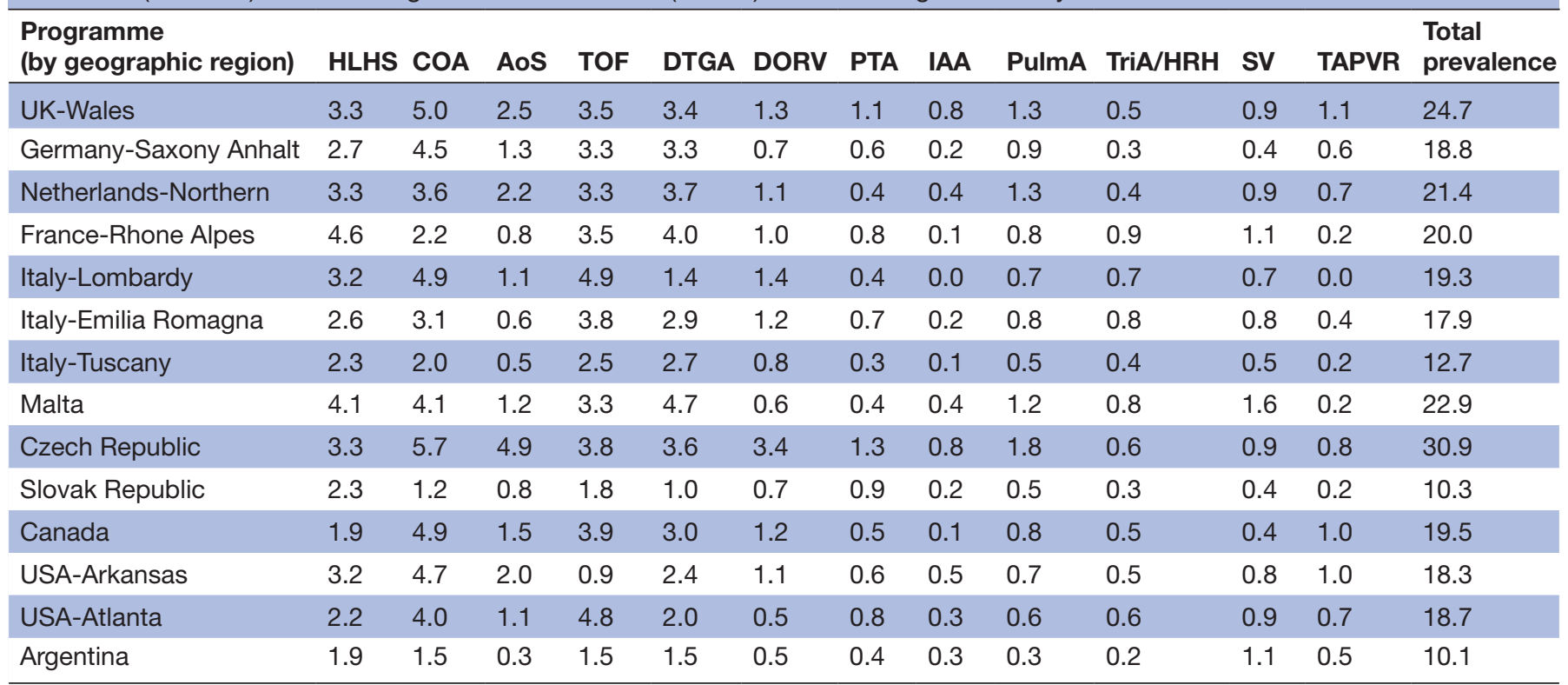

*ICBDSR programmes contributed data for different years within this time period (see table 1).

AoS, aortic valve stenosis; CCHD, critical congenital heart defects; COA, coarctation of the aorta; DTGA, d-transposition of great arteries; DORV, double outlet right ventricle; HLHS, hypoplastic left heart syndrome; IAA, interrupted aortic arch; PulmA, pulmonary valve atresia with intact ventricular septum; PTA, persistent truncus arteriosus; SV, single ventricle; TOF, tetralogy of Fallot; TriA/HRH, tricuspid valve atresia/hypoplastic right heart; TAPVR, total anomalous pulmonary venous return.

(10.3 and 10.1 per 10000 births, respectively, table 2 and figure 1). The highest LB prevalence among all programmes was observed in Malta (22.4 per 10000 ). During the study period, CCHD showed an increasing trend in total prevalence in France-Rhone Alpes and USA-Arkansas, a decreasing trend in the Czech Republic and USA-Atlanta, and more complex trends in Northern Netherlands and Germany-Saxony Anhalt (online supplementary table S1).

The difference between total and LB prevalence of CCHD (figure 1) reflected the proportion of TOPFA cases (table 3). The proportion of TOPFA cases varied several-fold in programmes in which TOPFA were legal, from $<1 \%$ in USA-Arkansas to $24 \%$ in the Czech Republic and 35\% in France-Rhone Alpes. In Malta and Argentina, termination of pregnancy is not allowed. In IndiaChennai, information on the outcome of pregnancy was unavailable in the majority of cases. The proportion of SB CCHD cases was small, on average $2 \%$ of total cases, with minor differences among programmes (highest SB proportion of $4 \%$ in Northern Netherlands).

Patterns and distribution of the 12 CCHD types

The total prevalence by CCHD type is presented by programme in table 2. Although the prevalence varied, the proportion of CCHD types was similar among programmes. Five CCHD types-HLHS, CoA and AoS (left ventricular outflow tract obstruction anomalies), TOF and DTGA-accounted for $71 \%$ of cases, with some variations among programmes $(80 \%$ in Lombardy and $56 \%$ in India, online supplementary table S2).

\section{Prenatal diagnosis}

There was considerable variation in proportion of CCHD identified via prenatal diagnosis among programmes (figure 2) from $87 \%$ in France-Rhone Alpes to $13 \%$ in Malta and Slovak Republic. In IndiaChennai, an exclusively prenatal diagnosis programme, all cases by design were prenatally diagnosed. In programmes with a high proportion of prenatally diagnosed CCHD cases, the proportion of LBs tended to be lower and the proportion of TOPFA higher. The converse was also true: the proportion of LB cases was higher in programmes with a low fraction of prenatally diagnosed cases.

In most programmes, the proportion of CCHD cases prenatally diagnosed increased considerably during the study period, in some cases several-fold (table 4). The proportion prenatally diagnosed also varied by type of CCHD. Such proportion was higher for HLHS and SV, which markedly affect ventricular morphology, and lower for dTGA, TAPVR and AoS, which affect ventricular morphology less markedly or frequently, thereby making prenatal detection more difficult. Among CCHD types, the fraction prenatally diagnosed varied considerably between programmes, but the rank order was similar (table 5). For example, the proportion of HLHS cases prenatally diagnosed varied from $24 \%$ in Slovak Republic to 95\% in France-Rhone Alpes and $100 \%$ in Italy-Lombardy, but within each programme, HLHS was the CCHD diagnosed prenatally most frequently. 


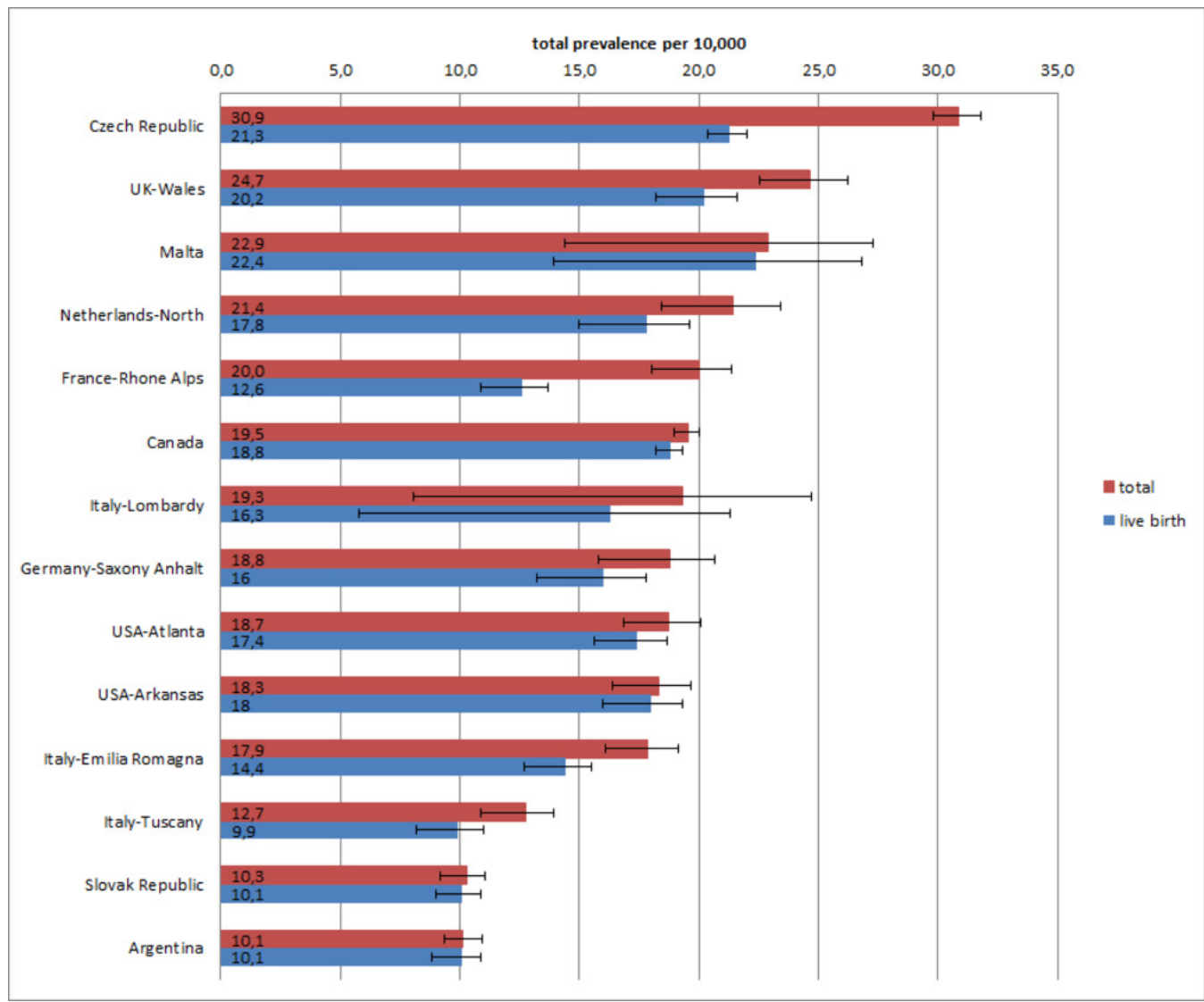

Figure 1 Total prevalence and live birth prevalence (per 10000 births) with 95\% Cls for 12 CCHD types, by programme, International Clearinghouse for Birth Defects Surveillance and Research (ICBDSR) Critical Congenital Heart Defects Prenatal Diagnosis study 2000-2014. ICBDSR programmes, ordered by descending total prevalence, contributed data for different years within this time period (see table 1).Chennai, India programme is not included in prevalence estimates because for this exclusively prenatal programme the denominator data (total births and total live births) are unavailable.

\section{Clinical presentation}

The proportion of prenatally detected cases was higher in syndromic and MCA CCHD cases compared with isolated cases, and the difference was more pronounced in programmes with lower overall prenatal detection proportion (figure 3). Overall, most CCHD present as isolated $(80 \%)$, with variations between programmes. In Italy-Tuscany and Czech Republic, 90\% of the CCHD cases presented as isolated, whereas in USA-Arkansas and USA-Atlanta, $68 \%$ presented as isolated (table 2). Some CCHD types were more commonly reported as isolated (AoS, DTGA, TRiA/HRH, HLHS and COA in $>80 \%$ of the cases) compared with others such as PTA and IAA, which had a higher proportion of syndromic cases $(>17 \%$ of the cases, data not shown).

\section{Mortality in first month of life}

Because of the variations in follow-up period among programmes, we focused the analysis on neonatal mortality (mortality by the first month of life in LBSs). The highest neonatal mortality was found in Argentina (25.5\%) and Malta $(24.1 \%)$ (figure 4). In these countries, termination of pregnancy is not allowed, and prenatal detection for CCHD is relatively low (table 5 and figure 2). The lowest neonatal mortality was found in Emilia Romagna (4.0\%),
Germany-Saxony Anhalt (5.4\%), Tuscany (7.8\%), UKWales (8.7\%), Czech Republic (9.6\%), Italy- Lombardy $(10.9 \%)$ and France-Rhone Alpes (11.1\%). In these programmes, TOPFA proportions are comparatively high (table 2).

\section{DISCUSSION}

In this retrospective cohort study of more than 18000 CCHD cases from 15 birth defect surveillance programmes from Europe, North and South America and Asia, we observed several remarkable patterns and trends in the occurrence and prenatal diagnosis of CCHD.

First, CCHDs are common regardless of geography and ascertainment programme. The median total prevalence was 19 per 10000 births, or approximately 1 in 500 births, similar to prior reports. ${ }^{13}$ However, total prevalence varied threefold among regions and programmes (figure 1). At least some and perhaps most of such variation is likely related to methodology, that is, the local capacity to detect and report these conditions. Such methodological factors include the ascertainment period after birth, ranging from days to years in the different programmes (table 1), and the ability to obtain a detailed diagnosis, both for the cardiac anomaly and extracardiac findings. For example, 
Table 3 Cases of CCHD by programme and by pregnancy outcome and clinical presentation, International Clearinghouse for Birth Defects Surveillance and Research (ICBDSR) Critical Congenital Heart Defects (CCHD) Prenatal Diagnosis study 2000$2014^{*}$

\begin{tabular}{|c|c|c|c|c|c|c|c|c|}
\hline \multirow[b]{2}{*}{ Programme - region } & \multirow{2}{*}{$\begin{array}{l}\text { Total } \\
\text { cases }\end{array}$} & \multicolumn{4}{|c|}{ Pregnancy outcome (\%) } & \multicolumn{3}{|c|}{ Clinical presentation (\%) } \\
\hline & & LB & SB & TOPFA & Unknown & Isolated & MCA & Syndromic \\
\hline UK-Wales & 1003 & 81.2 & 2.5 & 16.4 & 0 & 71.6 & 15.5 & 13.0 \\
\hline Germany-Saxony Anhalt & 392 & 84.7 & 2.0 & 13.3 & 0 & 74.7 & 14.0 & 11.2 \\
\hline Netherlands-Northern & 477 & 82.4 & 4.2 & 13.4 & 0 & 74.8 & 11.9 & 13.2 \\
\hline France-Rhone Alps & 820 & 61.7 & 3.2 & 35.1 & 0 & 70.0 & 17.6 & 12.4 \\
\hline Italy-Emilia Romagna & 795 & 79.5 & 0.1 & 20.4 & 0 & 81.3 & 9.4 & 9.3 \\
\hline Italy-Lombardy & 55 & 83.6 & 3.6 & 12.7 & 0 & 85.5 & 7.3 & 7.3 \\
\hline Italy-Tuscany & 451 & 77.2 & 2.2 & 20.6 & 0 & 90.5 & 4.7 & 4.9 \\
\hline Malta & 111 & 97.3 & 2.7 & na & 0 & 79.3 & 9.0 & 11.7 \\
\hline Czech Republic & 4569 & 68.4 & 0.8 & 23.6 & 7.3 & 89.6 & 5.8 & 4.6 \\
\hline Slovak Republic & 687 & 98.1 & 0.4 & 1.2 & 0.3 & 83.6 & 10.9 & 5.5 \\
\hline Canada & 6157 & 95.2 & 1.7 & 3.1 & 0 & 79.2 & 11.6 & 9.1 \\
\hline USA-Arkansas & 722 & 97.4 & 2.1 & 0.4 & 0.1 & 67.6 & 20.2 & 12.2 \\
\hline USA-Atlanta & 796 & 92.8 & 2.9 & 3.4 & 0.9 & 67.5 & 13.7 & 18.8 \\
\hline Argentina & 609 & 98.4 & 1.5 & na & 0.2 & 75.5 & 18.4 & 6.1 \\
\hline India-Chennai† & 599 & 6.8 & 0.7 & 35.2 & 57.3 & 82.8 & 15.4 & 1.8 \\
\hline
\end{tabular}

${ }^{*}$ ICBDSR programmes contributed data for different years within this time period (see table 1).

†India-Chennai is a prenatal programme, and only includes congenital heart defects that are prenatally diagnosed

CCHD, critical congenital heart defects; LB, live births; MCA, multiple congenital anomalies; na, not available; SB, stillbirths; TOPFA, termination of pregnancy for fetal anomaly.

programmes reporting the lowest prevalence rates (Slovak Republic and Argentina) have a short postnatal ascertainment period (at birth/hospital discharge). Also, with few exceptions, programmes with low prevalence rates tend to report few syndromic CCHD cases (table 2). A further factor is a programme's ability to ascertain and record terminations of affected pregnancies (table 2). In countries where terminations of pregnancy are illegal, no terminations are recorded. However, in countries where terminations are legal, a reliable surveillance system may not be able to include these events, and they will be under-reported in these data. Part of the variation in

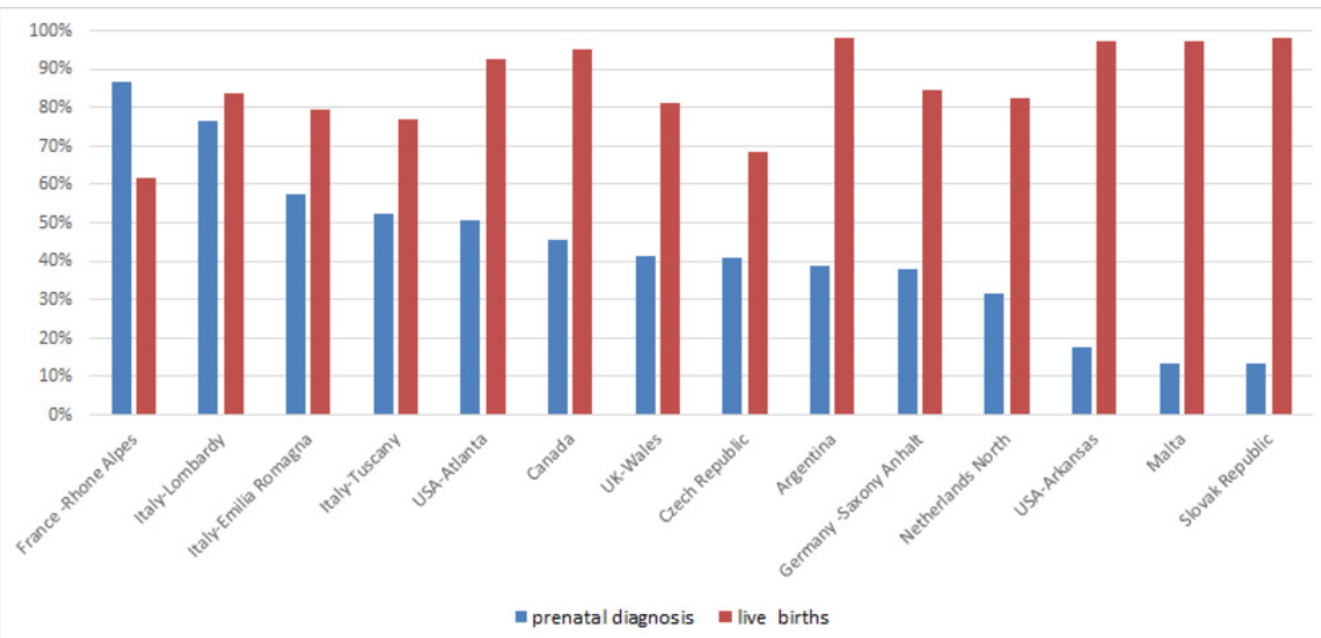

Figure 2 Proportion prenatally diagnosed and proportion of live births among all CCHD cases by programme, International Clearinghouse for Birth Defects Surveillance and Research (ICBDSR) Critical Congenital Heart Defects (CCHD) Prenatal Diagnosis study 2000-2014. ICBDSR Programmes (ordered by descending prenatal diagnosis proportion) contributed data for different years within this time period (see table 1). India-Chennai is not included in the figure because as an exclusively prenatal diagnosis programme, all cases by design were prenatally diagnosed, and information on outcome of pregnancy is missing in the majority of cases. 


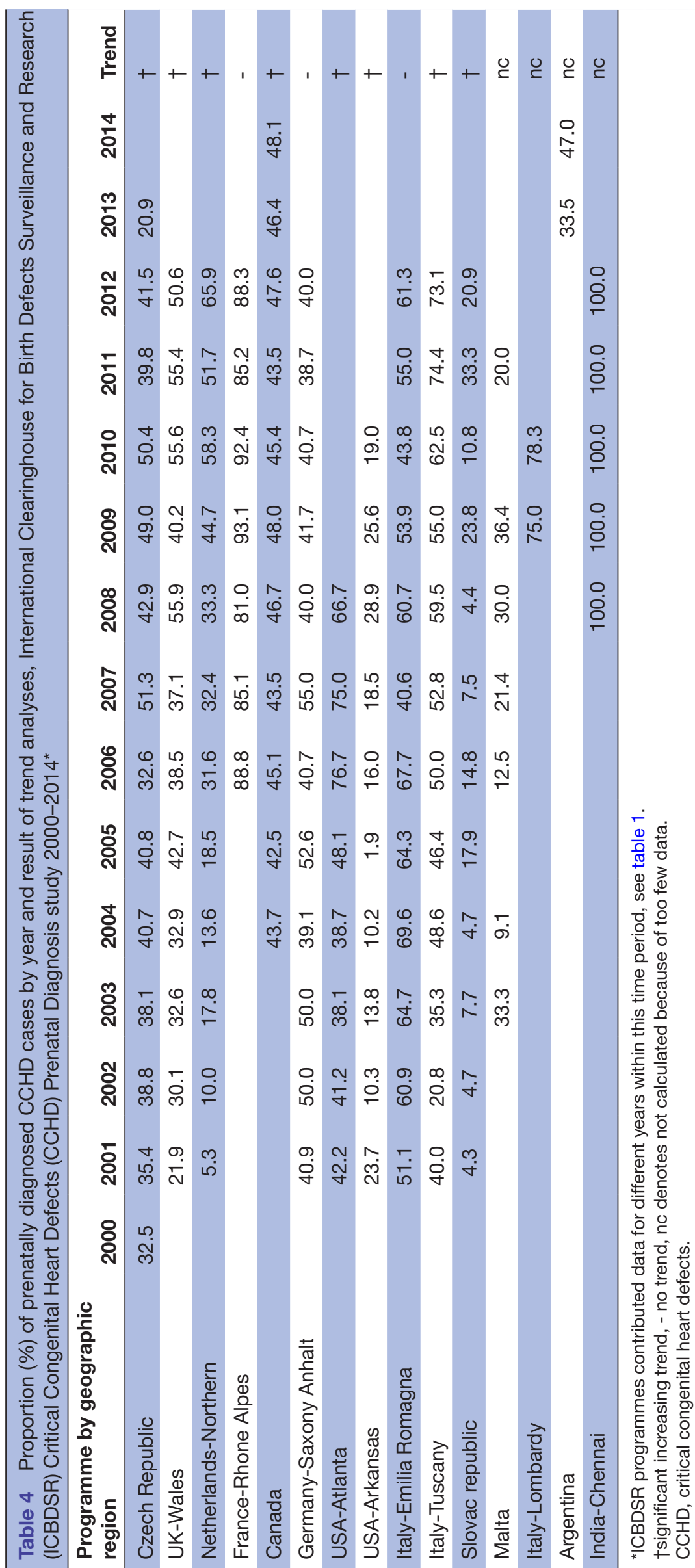




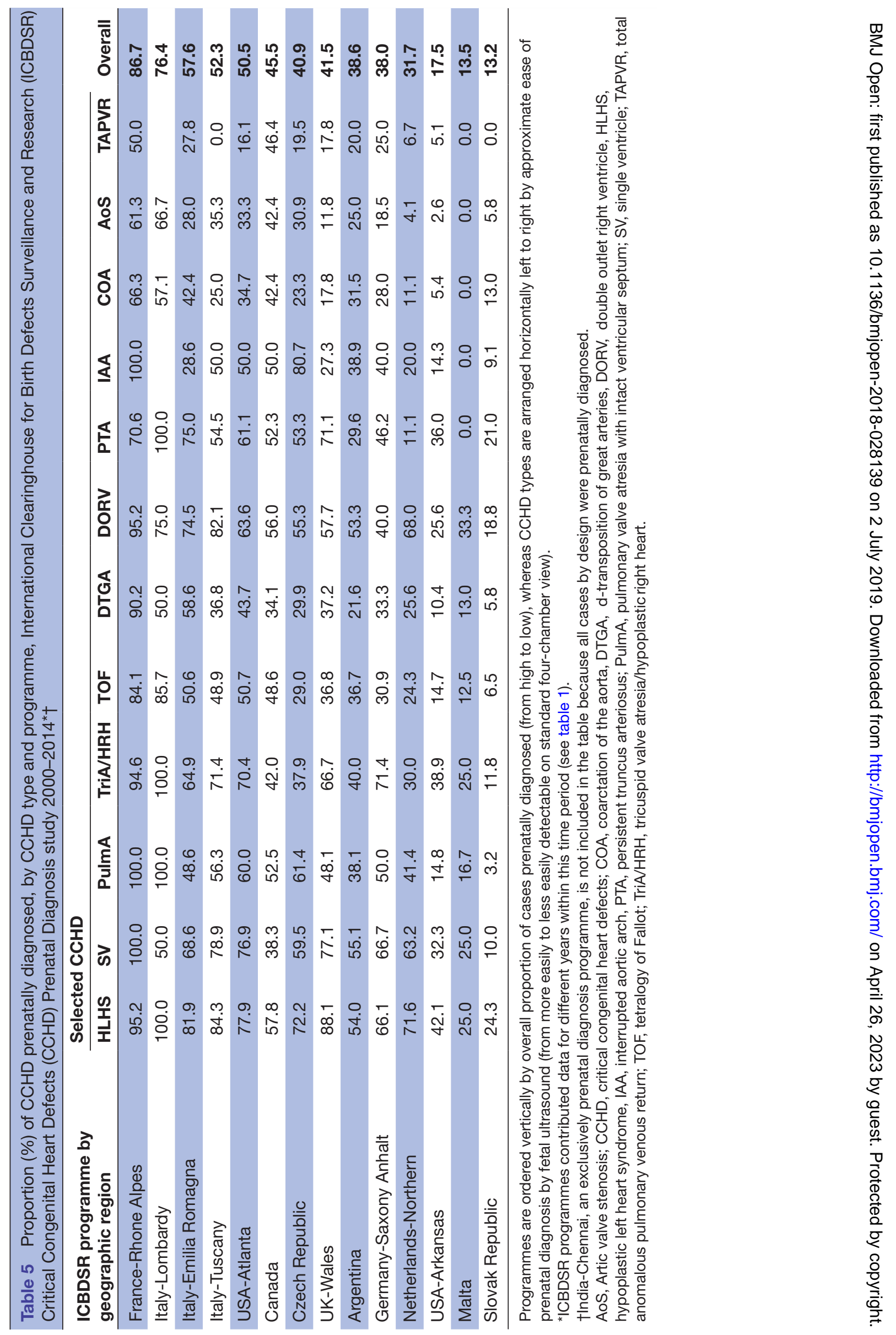




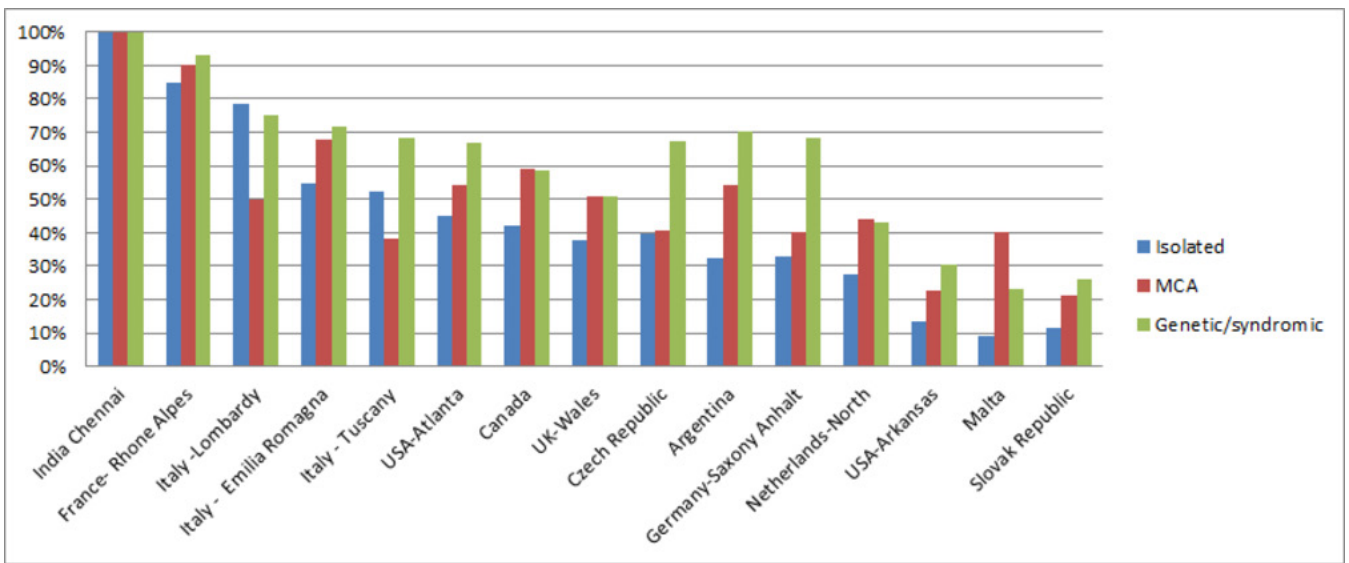

Figure 3 Proportion of prenatally diagnosed CCHD cases according to clinical presentation and by programme International Clearinghouse for Birth Defects Surveillance and Research (ICBDSR) Critical Congenital Heart Defects (CCHD) Prenatal Diagnosis study 2000-2014. Programmes (ordered by descending prenatal detection proportion) contributed data for different years within this time period (see table 1). India-Chennai is a prenatal diagnosis-only programme. MCA, multiple congenital anomalies.

prevalence could reflect true geographical differences in CCHD occurrence due to either genetic predisposition or the frequency of risk factors such as pre-existing maternal diabetes, maternal obesity, use of teratogenic drugs and smoking. ${ }^{18-22}$

A second finding was that, whereas the total prevalence varied considerably among programmes, the relative distribution of CCHD types was similar. For example, HLHS, CoA, TOF and DTGA were consistently among the most prevalent CCHD (online supplementary table S2). The exception was India-Chennai, which deviated from the other programmes likely because of the exclusively prenatal nature of that programme.

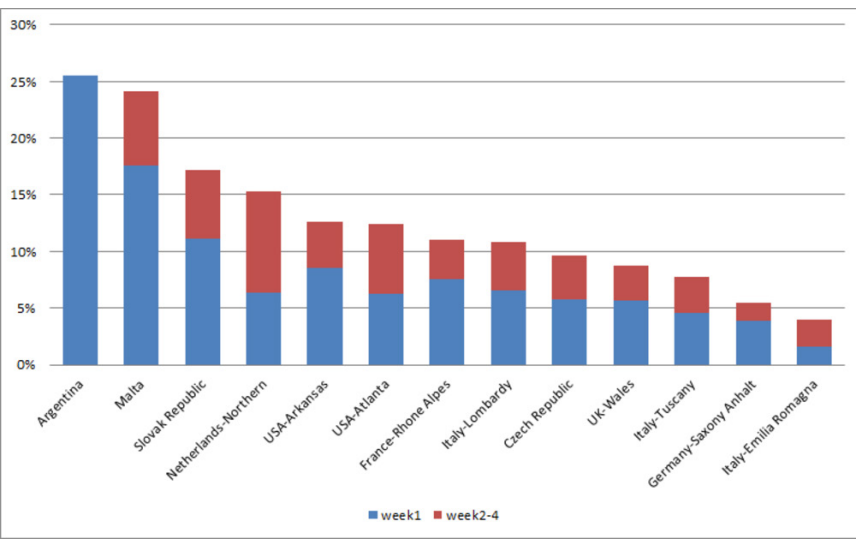

Figure 4 First month mortality in live birth cases with selected CCHD by programme, International Clearinghouse for Birth Defects Surveillance and Research (ICBDSR) Critical Congenital Heart Defects (CCHD) Prenatal Diagnosis study 2000-2014. ICBDSR programmes (ordered by descending first month mortality) contributed data for different years within this time period (see table 1). India-Chennai and Canada are not included in the graph: pregnancy outcomes in India-Chennai are poorly reported and Canada reported on mortality 1 year after birth, not specified in first week or first month mortality.
A third notable finding was the variation and patterns of prenatal detection (table 5). Although in all regions second trimester scans are offered as part of standard obstetric care, prenatal detection by programme varied from $13 \%$ in Slovak Republic to $87 \%$ in France-Rhone Alpes, suggesting a role of policies, technical expertise, scanning protocols and practice related to prenatal screening. Even the two programmes in the southeastern USA, Arkansas and Atlanta, Georgia, had widely disparate prenatal detection proportions. The difference in prenatal detection of CCHD between these two programmes is consistent with previous reports, which have shown geographic variations in the USA, ranging from $11.8 \%$ to $53.4 \% .^{23}$ Prenatal detection was more frequent for clinically complex cases (eg, those with a syndrome or multiple congenital anomalies). This finding, reported also in other studies, ${ }^{15} 24$ likely reflects a greater intensity of fetal examination when any anomaly is identified prenatally. Prenatal detection was also higher for CCHD with primary or significant involvement of the ventricles, such as HLHS and SV, compared with CCHD in which either additional outflow tract views on fetal ultrasound are required (eg, DTGA) or the defects are objectively harder to identify (eg, TAPVR, CoA and AoS). In addition, other studies have suggested that a postnatal diagnosis is more common for CCHD that require a view on fetal ultrasound other than a four-chamber view, lesions that are isolated (eg, absence of another organ system anomaly) or in a setting of poverty or lower population density community. ${ }^{25}$ These findings taken together highlight the crucial role of policies, training and access in driving the rates of prenatal diagnosis in the population.

The proportion of prenatally detected CCHD cases significantly increased over time in most programmes (table 4). The specific patterns varied among programmes. For example, in the Northern Netherlands, a sharp increase in prenatal detection coincided with 
the introduction of the prenatal screening programme in 2007 (including a 20 week anomaly scan), ${ }^{26} 27$ whereas in other programmes, the increase was more gradual. Increasing trends in prenatal diagnosis were also observed in other studies ${ }^{232-31}$ and have been variably attributed to improvements in ultrasound technology as well as policies and recommendations pertaining to examination of the fetal anatomy. ${ }^{32-34}$ For example, in 2006, the International Society for Ultrasound in Obstetrics and Gynaecology issued a guideline that recommended adding the outflow tract view to the basic four-chamber view. ${ }^{35}$

We examined the patterns of prenatal diagnosis in relation to TOPFA proportions. In programmes where such terminations are legal, TOPFA occurred in less than $1 \%-35 \%$ of CCHD cases. Two patterns seemed to emerge. In some programmes such as USA-Arkansas and Slovak Republic, low TOPFA proportions co-occur with a low proportion of prenatal diagnosis, and second, clinically complex cases (eg, associated with other extracardiac anomalies or syndromic cases) seemed to be prenatally diagnosed more often (figure 3), though the relation between clinical complexity and TOPFA was less clear. Pregnancy outcome is not a direct function of prenatal diagnosis. For example, factors that can influence the TOPFA proportion after prenatal diagnosis may be social or cultural (for instance acceptance of TOPFA) and include the legal gestational age limit for pregnancy termination and the extent to which TOPFA are reported to or captured in the healthcare databases.

Finally, neonatal mortality also varied regionally. The study did not specifically assess the system or personal factors potentially associated with such variation, such as gestational age at birth or birth weight. However, we noted that the neonatal mortality was highest in Malta and Argentina where termination of pregnancy is not allowed and prenatal detection of CCHD is low. The lowest neonatal mortality was found in countries where the TOPFA proportions were highest. These findings, though not conclusive, suggest two possibilities. First, prenatal detection might help improve the care of babies with CCHD by allowing for a better plan of care at birth when compared with the unanticipated urgency at birth if no prenatal diagnosis was made. ${ }^{12} 13$ Second, terminations of pregnancy may disproportionately include the anatomically more severe cases (even within the same CCHD type), such that the overall survival is skewed towards what might be only an apparent improvement in outcomes. ${ }^{36}$

\section{Strengths and weaknesses of the study}

The study has several strengths, including the large sample of the CCHD cohort (>18000 cases) and the systematic nature of case ascertainment whether through population-based or hospital-based programmes. Including programmes from areas with different policies and healthcare systems allowed us a wider view of the inter-related factors that can influence reported prevalence, ascertainment and prenatal diagnosis. Programmes submitted individual case records that were centrally reviewed by clinicians with expertise in genetics (LDB and JEHB) and paediatric cardiology (LDB). This review aimed at harmonising the CCHD diagnoses (eg, cases with more than one CCHD code were systematically assigned a primary diagnosis) as well as the clinical classification as isolated, MCA or syndromic case. The study also has limitations. The quality and completeness of the data submitted centrally depends on the programme's methods related to data collection, coding and classification (eg, the degree to which clinical staff is involved in these processes). Also, we did not have details on the severity of each CCHD case, which may have contributed to variation across programmes. For example, the clinical presentation of lesions such as AoS and COA can range from mild (eg, not readily identifiable prenatally or clinically at birth) to severe (eg, a truly critical condition in the neonatal period). These variations would influence a programme's ability to detect these conditions early in life or prenatally and would therefore affect findings such as the total prevalence and the proportion of cases prenatally diagnosed. A last limitation is the challenge and variability in ascertainment of pregnancies that ended in a termination.

\section{Meaning of the study: possible mechanisms and implications} for clinicians or policy makers

Ultimately, these findings, together with prior reports from the literature, have both public health and clinical implications for the care and prevention of CCHD. First, the high prevalence ( 1 in 500 births) underscores the universal need to address primary prevention and care of CCHD aggressively. Care in particular could be enhanced with earlier diagnosis. In this regard, prenatal diagnosis can complement pulse oximetry newborn screening, and compared with the latter, allow for more time and hence more thoughtful management decisions by well-informed families and clinicians. ${ }^{3738}$

The increasing trends in prenatal diagnosis rates also highlight the potential for significant changes in the epidemiology and clinical outcomes of CCHD. Although the magnitude of these trends vary in the included programmes, the potential implications are vast. Prenatal diagnosis may continue to influence the reported prevalence at birth as well as the outcomes (eg, morbidity, survival) by a combination of more complete and timely detection and, to a varying extent, its influence on rates of TOPFA. The results of this study demonstrate the value of ongoing surveillance of CCHD in this changing environment.

Tracking and evaluating the patterns of CCHD occurrence is also important in the quest to discover the causes of these severe conditions. For example, in aetiological studies, it is particularly important to include all affected fetuses, as SBs and terminations of pregnancy are more likely to be over-represented in more severe cases. Failing to include such cases would limit the range and possibly skew the findings.

Finally, ongoing monitoring of the CCHD cohort, from pregnancy onwards, is important for researchers to 
appropriately evaluate long-term outcomes and track the burden of disease on population health.

Important questions remain. Is prenatal diagnosis improving population health? In an era of improving (and often more costly) diagnostic technology, are current systems increasing rather than eliminating potential health disparities? Are we providing the most current information about occurrence and outcomes to clinicians and families for appropriate counselling in the presence of a prenatally detected CCHD? Answering such questions requires a joint effort of epidemiologists and clinicians generating high-quality information and tracking such data over time. Leveraging existing programmes, data sharing and central clinical review and analysis may enhance efficiencies and inform these questions. International networks such as the ICBDSR, the National Birth Defects Prevention Network, and EUROCAT European surveillance of congenital anomalies can help provide the data, the analytic capacity and a long-term vision for sustained, accurate and timely monitoring of the health impact of CCHD, as a basis for interventions aimed at improving primary prevention and care.

\section{Author affiliations}

${ }^{1}$ Department of Genetics, Eurocat registration Northern Netherlands, University of Groningen, University Medical Center Groningen, Groningen, The Netherlands ${ }^{2}$ Division of Medical Genetics, Department of Pediatrics, University of Utah, Salt Lake City, Utah, USA

${ }^{3}$ Registre Des Malformations en Rhone Alpes, REMERA, Lyon, France

${ }^{4}$ Neonatology Unit, S.Orsola-Malpighi Hospital, Department of Medical and Surgical Sciences, University of Bologna, Bologna, Italy

${ }^{5}$ Metropolitan Atlanta Congenital Defects Program, National Center on Birth Defects and Developmental Disabilities, Centers for Disease Control and Prevention, Atlanta, Georgia, USA

${ }^{6}$ Malta Congenital Anomalies Registry, Directorate for Health Information and Research, Malta, Malta

${ }^{7}$ National Network of Congenital Anomalies of Argentina (RENAC), National Center of Medical Genetics, National Ministry of Health, Buenos Aires, Argentina

${ }^{8}$ Maternal, Child and Youth Health Division, Public Health Agency of Canada, Ottawa, Canada

${ }^{9}$ Arkansas Reproductive Health Monitoring System, University of Arkansas for Medical Sciences, Fay W Boozman College of Public Health and the Arkansas Children's Research Institute, Little Rock, Arkansas, USA

${ }^{10}$ Institute of Clinical Physiology, National Research Council and Fondazione Toscana Gabriele Monasterio, Tuscany Registry of Congenital Defects, Pisa, Italy

${ }^{11}$ Malformation Monitoring Centre, Medical Faculty, Otto von Guericke University, Magdeburg, Germany

${ }^{12}$ Birth Defects Registry of India, Mediscan Systems, Chennai, India

${ }^{13}$ Institute of Medical Biology and Genetics First Faculty of Medicine Charles

University and General University Hospital, Prague, Czech Republic

${ }^{14}$ Slovak Teratologic Information Centre (FPH), Slovak Medical University, Bratislava, Slovakia

${ }^{15}$ Lombardy Birth Defects Registry, Fondazione IRCCS Instituto Nazionale Tumori, Milan, Italy

${ }^{16}$ Congenital Anomaly Register and Information Service for Wales, Public Health Wales, Swansea, Wales, UK

${ }^{17}$ International Center on Birth Defects, University of Utah, Salt Lake City, Utah, USA

Acknowledgements We would like to thank each International Clearinghouse for Birth Defect Surveillance and Research member programme's staff for providing and processing the information on the cases with critical congenital heart defects and on the characteristics of their program.
Contributors LDB conceived the study. MKB and LDB developed the protocol and supervised the study. MKB, LDB and SK conducted the data analysis. LDB and JEHB reviewed the clinical classification of cases. MKB and LDB wrote the first draft of the article. EA, GC, JC, HEKdW, MG, BG, SL, WNN, AP, AR, SC, AS, ES, GT, DT and PM curated and submitted the case data from their programs. All coauthors made substantial contributions to the conduct of the study, interpretation of results and critical revisions of the manuscript. Programme directors are guarantors of the integrity of the data submitted for central analysis. MKB and LDB are overall guarantors.

Funding The programmes participating in this study may receive local funding. The programme in Czech Republic is supported by Ministry of Health of the Czech Republic, grant nr. AZV 17-29622A.

Disclaimer The findings and conclusions in this report are those of the authors and do not necessarily represent the official position of the Centers for Disease Control and Prevention.

Competing interests None declared.

Patient consent for publication Not required.

Provenance and peer review Not commissioned; externally peer reviewed. Data sharing statement No additional data are available.

Open access This is an open access article distributed in accordance with the Creative Commons Attribution 4.0 Unported (CC BY 4.0) license, which permits others to copy, redistribute, remix, transform and build upon this work for any purpose, provided the original work is properly cited, a link to the licence is given, and indication of whether changes were made. See: https://creativecommons.org/ licenses/by/4.0/.

\section{REFERENCES}

1. Dolk H, Loane M, Garne E, et al. Congenital heart defects in Europe: prevalence and perinatal mortality, 2000 to 2005 . Circulation 2011;123:841-9.

2. Reller MD, Strickland MJ, Riehle-Colarusso T, et al. Prevalence of congenital heart defects in metropolitan Atlanta, 1998-2005. J Pediatr 2008;153:807-13.

3. Oster ME, Lee KA, Honein MA, et al. Temporal trends in survival among infants with critical congenital heart defects. Pediatrics 2013;131:1502-.

4. Mackie AS, Tran DT, Marelli AJ, et al. Cost of Congenital Heart Disease Hospitalizations in Canada: A Population-Based Study. Can $J$ Cardiol 2017;33:792-8.

5. Arth AC, Tinker SC, Simeone RM, et al. Inpatient Hospitalization Costs Associated with Birth Defects Among Persons of All Ages United States, 2013. MMWR Morb Mortal Wkly Rep 2017;66:41-6.

6. Boneva RS, Botto LD, Moore CA, et al. Mortality associated with congenital heart defects in the United States: trends and racial disparities, 1979-1997. Circulation 2001;103:2376-81.

7. Gilboa SM, Salemi JL, Nembhard WN, et al. Mortality resulting from congenital heart disease among children and adults in the United States, 1999 to 2006. Circulation 2010;122:2254-63.

8. Jortveit J, Eskedal L, Hirth A, et al. Sudden unexpected death in children with congenital heart defects. Eur Heart J 2016;37:621-6.

9. Knowles RL, Bull C, Wren C, et al. Mortality with congenital heart defects in England and Wales, 1959-2009: exploring technological change through period and birth cohort analysis. Arch Dis Child 2012;97:861-5

10. Blyth M, Howe D, Gnanapragasam J, et al. The hidden mortality of transposition of the great arteries and survival advantage provided by prenatal diagnosis. BJOG 2008;115:1096-100.

11. Bonnet $D$, Coltri $A$, Butera $G$, et al. Detection of transposition of the great arteries in fetuses reduces neonatal morbidity and mortality. Circulation 1999;99:916-8.

12. Holland BJ, Myers JA, Woods CR. Prenatal diagnosis of critical congenital heart disease reduces risk of death from cardiovascular compromise prior to planned neonatal cardiac surgery: a metaanalysis. Ultrasound Obstet Gynecol 2015;45:631-8.

13. Morris SA, Ethen MK, Penny DJ, et al. Prenatal diagnosis, birth location, surgical center, and neonatal mortality in infants with hypoplastic left heart syndrome. Circulation 2014;129:285-92.

14. Tworetzky W, McElhinney DB, Reddy VM, et al. Improved surgical outcome after fetal diagnosis of hypoplastic left heart syndrome. Circulation 2001;103:1269-73. 
15. Pinto NM, Keenan HT, Minich LL, et al. Barriers to prenatal detection of congenital heart disease: a population-based study. Ultrasound Obstet Gynecol 2012;40:418-25.

16. Clur SA, Bilardo CM. Early detection of fetal cardiac abnormalities: how effective is it and how should we manage these patients? Prenat Diagn 2014;34:1235-45.

17. Egbe A, Uppu S, Lee S, et al. Changing prevalence of severe congenital heart disease: a population-based study. Pediatr Cardiol 2014;35:1232-8.

18. Gilboa SM, Correa A, Botto LD, et al. Association between prepregnancy body mass index and congenital heart defects. Am J Obstet Gynecol 2010;202:e1-e10.

19. Baardman ME, Kerstjens-Frederikse WS, Corpeleijn E, et al. Combined adverse effects of maternal smoking and high body mass index on heart development in offspring: evidence for interaction? Heart 2012;98:474-9.

20. Hoang TT, Marengo LK, Mitchell LE, et al. Original findings and updated meta-analysis for the association between maternal diabetes and risk for congenital heart disease phenotypes. Am J Epidemiol 2017;186:118-28.

21. Jenkins KJ, Correa A, Feinstein JA, et al. Noninherited risk factors and congenital cardiovascular defects: current knowledge: a scientific statement from the American Heart Association Council on Cardiovascular Disease in the Young: endorsed by the American Academy of Pediatrics. Circulation 2007;115:2995-3014.

22. Mills JL, Troendle J, Conley MR, et al. Maternal obesity and congenital heart defects: a population-based study. Am J Clin Nutr 2010;91:1543-9.

23. Quartermain MD, Pasquali SK, Hill KD, et al. Variation in prenatal diagnosis of congenital heart disease in infants. Pediatrics 2015;136:e378-85.

24. Wong SF, Chan FY, Cincotta RB, et al. Factors influencing the prenatal detection of structural congenital heart diseases. Ultrasound Obstet Gynecol 2003;21:19-25.

25. Hill GD, Block JR, Tanem JB, et al. Disparities in the prenatal detection of critical congenital heart disease. Prenat Diagn 2015;35:859-63.

26. Baardman ME, du Marchie Sarvaas GJ, de Walle HE, et al. Impact of introduction of 20-week ultrasound scan on prevalence and fetal and neonatal outcomes in cases of selected severe congenital heart defects in The Netherlands. Ultrasound Obstet Gynecol 2014;44:58-63.
27. van Velzen CL, Clur SA, Rijlaarsdam ME, et al. Prenatal detection of congenital heart disease--results of a national screening programme. BJOG 2016;123:400-7.

28. Chew $\mathrm{C}$, Stone S, Donath SM, et al. Impact of antenatal screening on the presentation of infants with congenital heart disease to a cardiology unit. J Paediatr Child Health 2006;42:704-8.

29. Corcoran S, Briggs K, O' Connor H, et al. Prenatal detection of major congenital heart disease - optimising resources to improve outcomes. Eur J Obstet Gynecol Reprod Biol 2016;203:260-3.

30. van Velzen CL, Ket JCF, van de Ven PM, et al. Systematic review and meta-analysis of the performance of second-trimester screening for prenatal detection of congenital heart defects. Int J Gynaecol Obstet 2018;140:137-45.

31. Lytzen R, Veijlstrup N, Bjerre J, et al. Live born major congenital heart disease in Denmark; incidence. detection rate and termination of pregnancy rate from 1996-2013, JAMA Cardiol 2018;3:829-37.

32. Komisar J, Srivastava S, Geiger M, et al. Impact of changing indications and increased utilization of fetal echocardiography on prenatal detection of congenital heart disease. Congenit Heart Dis 2017:12:67-73.

33. Oggè $G$, Gaglioti $P$, Maccanti $S$, et al. Prenatal screening for congenital heart disease with four-chamber and outflow-tract views: a multicenter study. Ultrasound Obstet Gynecol 2006;28:779-84.

34. Sklansky MS, Berman DP, Pruetz JD, et al. Prenatal screening for major congenital heart disease: superiority of outflow tracts over the 4-chamber view. J Ultrasound Med 2009;28:889-99.

35. International Society of Ultrasound in Obstetrics \& Gynecology. Cardiac screening examination of the fetus: guidelines for performing the 'basic' and 'extended basic' cardiac scan. Ultrasound Obstet Gynecol 2006;27:107-13.

36. Boyle B, Addor MC, Arriola L, et al. Estimating Global Burden of Disease due to congenital anomaly: an analysis of European data. Arch Dis Child Fetal Neonatal Ed 2018;103:F22-8.

37. Bratt EL, Järvholm S, Ekman-Joelsson BM, et al. Parent's experiences of counselling and their need for support following a prenatal diagnosis of congenital heart disease--a qualitative study in a Swedish context. BMC Pregnancy Childbirth 2015;15:171.

38. Thakur V, Dutil N, Schwartz SM, et al. Impact of prenatal diagnosis on the management and early outcome of critical duct-dependent cardiac lesions. Cardiol Young 2018;28:548-53. 